\title{
Clinical management of patients with advanced Parkinson's disease treated with continuous intestinal infusion of levodopa/carbidopa
}

Diego Santos García ${ }^{*}$, Juan Carlos Martínez Castrillo², Víctor Puente Périz ${ }^{3}$, Agustín Seoane Urgorri" ${ }^{4}$ Servando Fernández Díez ${ }^{5}$, Vicente Benita León ${ }^{6}$, Beatriz Udaeta Baldivieso 7 , Antonia Campolongo Perillo ${ }^{8,9}$ \& Natividad Mariscal Pérez ${ }^{10}$

\section{Practice points}

- Treatment with levodopa-carbidopa intestinal gel (LCIG; Duodopa ${ }^{\oplus}$, AbbVie, IL, USA) has demonstrated both efficacy and effectiveness in improving motor complications, motor status, nonmotor symptoms and patients' quality of life.

- Candidate selection must be individualized and should be considered in patients with disabling motor complications who want to be treated and who have neither label contraindications nor serious cognitive, behavioral or emotional complications.

- Old age and higher degree of neuropsychiatric impairment are predictors of poor response and higher risk of therapy discontinuation.

- Although the time since disease onset does not influence the outcome, we recommend to avoid unnecessary delay in the start of LCIG therapy, since this therapy provides better control of motor or nonmotor symptoms and improves patient's quality of life, compared with the best conventional therapy (oral medication).

- Compared with other treatments of advanced Parkinson's disease, selection criteria for LCIG are less stringent, and patients with contraindications for deep brain stimulation or apomorphine infusions can show a good response to LCIG therapy.

- We recommend establishing and following a protocol or clinical management guidelines for candidate selection, treatment implementation and follow-up.

- Patient management should be multidisciplinary, with recommended involvement of neurologists, gastroenterologists and specialized nurses.

- Given that minor complications are common and that they may lead to serious complications, their early identification and proper management is essential.

- Managing LCIG therapy is relatively complex and requires previous learning.

'Section of Neurology, Hospital Arquitecto Marcide, Ferrol University Hospital Complex (CHUF), Ferrol, Spain

2Department of Neurology, Hospital Ramón y Cajal, Instituto Ramón y Cajal de Investigación Sanitaria, Madrid, Spain

${ }^{3}$ Department of Neurology, Hospital del Mar, Barcelona, Spain

${ }^{4}$ Section of Gastrointestinal Endoscopy, Department of Gastroenterology, Hospital del Mar, Barcelona, Spain

${ }^{5}$ Department of Gastroenterology, Hospital Clínico San Carlos, Madrid, Spain

${ }^{6}$ Department of Gastroenterology, Hospital Ramón y Cajal, Madrid, Spain

'Unit of Movement Disorders \& Parkinson's Disease, Gran Canaria University Hospital, Las Palmas de Gran Canaria, Spain

${ }^{8}$ Department of Neurology, Movement Disorders Unit, Hospital de la Santa Creu i Sant Pau, Universitat Autònoma de Barcelona,

Barcelona, Spain

9Department of Health Sciences, Universitat Oberta de Catalunya (UOC), Barcelona, Spain

${ }^{10}$ Nursing Clinic, Department of Neurology, Burgos University Hospital, Burgos, Spain

*Author for correspondence: diegosangar@yahoo.es

Future Medicine ${ }_{\text {part of }}$ 
Patients with Parkinson's disease often have a good initial response to dopaminergic therapy but later usually develop motor fluctuations and dyskinesia. In these patients, continuous infusion of levodopa-carbidopa intestinal gel (LCIG) allows for maintaining adequate dopamine levels and for improving motor and nonmotor symptoms, as well as quality of life and autonomy. Adequate candidate selection and follow-up are crucial for treatment success. Management should be multidisciplinary, and patient and caregiver education is a priority. This expert consensus document has been developed by a team of neurologists, gastroenterologists and nurses who have a vast experience in LCIG therapy, with an intention to provide knowledge and tools to facilitate patient management throughout all phases of LCIG treatment process.

First draft submitted: 17 February 2016; Accepted for publication: 15 March 2016; Published online: 14 April 2016

Levodopa-carbidopa intestinal gel (LCIG) is a combination of levodopa and carbidopa monohydrate (in a 4:1 ratio) in a gel that is indicated for the treatment by continuous intestinal infusion of patients with advanced levodopa-responsive Parkinson's disease (PD) with severe motor fluctuations and hyperkinesia or dyskinesia when available combinations of Parkinson medicinal products have not given satisfactory results [1].

LCIG enteral infusion decreases motor fluctuations and increases 'on' time in patients with advanced PD previously given oral treatment with levodopa associated to a dopa decarboxylase inhibitor. Motor fluctuations and dyskinesia are reduced because plasma levodopa levels remain stable within the therapeutic range [2]. The effect on motor fluctuations and dyskinesia is often achieved during the first day of treatment $[3,4]$. In addition to improving motor symptoms and/or complications ('off' duration, 'off' severity, 'on' duration, 'on' quality, time with disabling dyskinesia) [2,5-33], LCIG has been shown to improve various nonmotor symptoms [34-36], patient's quality of life $[37,38]$ and autonomy in daily activities [10,16,24-25,27].

The gel should be administered by means of a portable pump directly into the duodenum or proximal jejunum through a percutaneous endoscopic gastrostomy (PEG) using an external transabdominal catheter and an internal intestinal catheter. Though severe complications are rare, minor device and gastrostomy related complications are common, and adequate knowledge to solve them is required.

\section{Rationale}

Implementation of LCIG treatment at a new site requires commitment, collaboration and proper training of all the professionals involved. Adequate LCIG management requires a multidisciplinary team including the following healthcare professionals:

\section{- A neurologist specialized in movement disorders;}

- A gastroenterologist trained in endoscopy;

- A nurse specialized in PD, neurology or endoscopy.

Successful implementation of LCIG treatment in new centers requires practical guidelines that would provide knowledge, experience and tools and decreased variability in clinical practice. The present guidelines go beyond the product information provided by the manufacturer; they are the result of several years of day-to-day experience of using LCIG therapy in patients with advanced PD. The authors hope that these recommendations would serve as a reference for professionals who work with patients treated with LCIG and help them in solving any complication or doubt that may arise.

\section{Methodology}

These guidelines have been prepared by a multidisciplinary team comprising three neurologists, three gastroenterologists and three nurses specialized in the treatment of advanced PD with LCIG, who based their recommendations on the available literature (indicated with references), the protocols of their work centers (coded as P) and their broad experience (coded as E) in treatment of these patients. The search was conducted in MEDLINE-PubMed (initial search in January 2013 and a complementary search in March 2016) using the following terms: Parkinson's disease and (duodopa or levodopa) and (infusion or gel). 


\section{Box 1. Selection criteria for levodopa-carbidopa intestinal gel therapy.}

\section{Inclusion criteria}

- Parkinson's disease (according to Hughes criteria of the United Kingdom Brain Bank [39] and/or Movement Disorder Society criteria) in an advanced stage with presence of disabling motor complications (motor fluctuations with or without dyskinesia) not controlled on standard oral medication

- Negative and significant impact of motor complications on the quality of life of the patient and/or his/her family (E)

- Assessment of the presence of some nonmotor symptoms (pain, depression, anxiety, fatigue, attention, gastrointestinal, urinary, among others) that may improve with medication is recommended (E) [6,14,20,31-34]

- Good response to oral levodopa, with some 'on' periods of quality. Daily 'off' time and motor status in 'off' should be known to justify the need for LCIG (P)

- Good social and family support (the caregiver must be able to handle the LCIG pump and to provide the care needed)

- Patients and relatives must accept the procedure by signing an informed consent document

Exclusion criteria

- Label contraindications [1]

- Associated severe comorbidity or reduced life expectancy

- Inability of the patient and/or the people around him/her to understand and follow the whole treatment process

- Exclusion criteria for PEG for any indication

- Severe coagulation disorder. Anticoagulation therapy is not a contraindication, but anticoagulant dose should be adjusted during and after the endoscopic procedure, and risk of gastrointestinal bleeding should be assessed [40]

- Advanced age (>80 years) is not a contraindication, but the increased risk of complications at older ages and with greater comorbidity must be taken into consideration [28,41,42]

- LCIG usage in patients with dementia is not recommended [43]; however, in patients with mild-to-moderate cognitive impairment indication should be individualized, and other aspects should be considered (functional status, family support, degree of autonomy, among others) [36,44]

- Severe neuropsychiatric symptoms (psychosis, impulse control disorder, major depression, punding, dopaminergic dysregulation syndrome, generalized anxiety disorder), which are limiting for the patient and his/her family and not adequately controlled (E). These do not represent an absolute contraindication, and the decision should be individualized because some patients may experience improvement in these symptoms $[14,45,46]$

- Disabling motor symptoms not responding to treatment with oral levodopa, such as absence of postural reflexes, gait freezing or frequent falls due to gait disorder during 'on' periods $[47,48]$

LCIG: Levodopa-carbidopa instestinal gel; PD: Parkinson's disease; PEG: percutaneous endoscopic gastrostomy.

\section{Results}

\section{- Patient selection phase}

Potential candidates to receive LCIG should be selected by a neurologist experienced in PD management. However, involvement of additional specialized staff (specialized nurse, gastroenterologist, psychologist, speech therapist) is sometimes helpful for taking decisions. Compliance with selection criteria, which are detailed in Box $1[1,6,14,20,28,31$ $34,36,39-48]$, is a priority consideration.

\section{Work-up of the patient-candidate for LCIG}

A detailed patient work-up (Box 2) [49] is required before deciding if he/she is eligible for LCIG treatment. A complete evaluation must be done to collect information about: motor complications; other complications and/or symptoms; aspects related to cognitive status, mood, and behavior, quality of life and degree of autonomy; impact on the family/caregiver. Based on this information, a decision should be made, together with the patient and his/her family, whether to proceed to LCIG treatment. Detailed information about candidate preparation, nursing procedures and patient/caregiver education can be found in [50].

Three basic conclusions should be reached:

- The patient needs the treatment (disabling daily 'off' time, disabling nonmotor symptoms, poor quality of life) and could benefit from LCIG treatment (optimal response to intraintestinally delivered levodopa);

- There are no contraindications for LCIG;

- There is a good family support despite the potentially high caregiver burden [51].

Once the decision is made, it is recommendable not to delay the beginning of the treatment, since LCIG offers clear benefits over conventional oral treatment [26]. 
Box 2. Work-up of patients-candidates to receive treatment with levodopa-carbidopa intestinal gel.

- General examination (including exact weight)

- Complete neurological examination

- Assessment of Parkinson's disease ${ }^{\dagger}$ :

- Motor symptoms:

- Motor complications: daily time in 'on' and 'off' state and 'with disabling dyskinesia' (diary and/or UPDRS-IV and/or MDS-UPDRS-IV and/or UDysRS)

- Motor stage (Hoehn \& Yahr scale) in 'off' and 'on'

- Motor severity (UPDRS-III or MDS-UPDRS in 'off' and 'on')

- Nonmotor symptoms: NMSS

Neuropsychological study:

- Cognitive assessment: MMSE, MoCA, PD-CRS

- Mood and/or anxiety: BDI, HADS

- Behavioral disorders: NPI

Quality of life: PDQ-39SI

- Degree of autonomy: ADLS

Calculation of the equivalent daily dose of oral levodopa (mg/day) [49]

- Caregiver evaluation:

- A good family support should be ensured

- Assessment of main caregiver status: ZCBI, CSI

- Supplemental tests:

- Laboratory tests including liver profile, ESR, complete blood count, chemistry and coagulation

Thyroid hormones

- Serum levels of vitamin B12 and folate

Others as applicable (homocysteine, $x$-rays, electrophysiological study, among others)

†Use of established scales may be beneficial for documentation of changes.

ADLS: Activities of daily living scale; BDI: Beck depression inventory; CSI: Caregiver strain index; ESR: Erythrocyte sedimentation rate; HADS: Hospital anxiety and depression scale; MDS: Movement disorder society; MMSE: Mini-mental state examination; MoCA: Montreal cognitive assessment; NMSS: Nonmotor symptom scale; NPI: Neuropsychiatric inventory; PD-CRS: Parkinson's disease cognitive rating scale; PDQ-39SI, 39-item Parkinson's disease questionnaire summary index score; UDysRS, Unified dyskinesia rating scale; UPDRS: Unified Parkinson disease rating scale; ZCBI: Zarit caregiver burden inventory.

Simplification of dopaminergic treatment

One of the advantages of LCIG is treatment simplification, so that many patients can receive monotherapy with levodopa/carbidopa [22]. It is recommended to adjust medication before starting LCIG to leave the patient on monotherapy and to carry out drug response and tolerability tests [52], though some studies suggest that a direct switch from polytherapy to LCIG monotherapy might be safe and effective [53]. Table 1 shows the conversion factors for calculating levodopa equivalent daily dose (LEDD) [49].

Table 1. Conversion factors for antiparkinsonian drugs to yield levodopa equivalent doses proposed by Tomlinson et al.

\begin{tabular}{|llll|}
\hline Drug & Dose, $\mathrm{mg}$ (example) & Conversion factor & Levodopa equivalent dose (mg) \\
\hline Immediate-release L-dopa & $400(100,1-1-1-1)$ & $\times 1$ & 400 \\
\hline Controlled-release L-dopa & $200(100,1-0-0-0-0-1)$ & $\times 0.75$ & 150 \\
\hline Entacapone (or Stalevo $\left.{ }^{\oplus}\right)$ & $600\left(\right.$ Stalevo $\left.^{\oplus} 200,1-1-1\right)$ & L-dopa $\times 0.33$ & $198(198+600=798)$ \\
\hline Tolcapone & $600(200,1-1-1)$ & L-dopa $\times 0.5$ & 300 \\
\hline Pramipexol & $3.15(2.01+1.05)$ & $\times 100$ & 315 \\
\hline Ropinirol & 24 & $\times 20$ & 480 \\
Rotigotine & 16 & $\times 30$ & 480 \\
\hline Selegiline & $10(5,1-1-0)$ & $\times 10$ & 100 \\
\hline Rasagiline & 1 & $\times 100$ & 100 \\
\hline Amantadine & $200(100,1-1-0)$ & $\times 1$ & 200 \\
\hline Data taken with permission from [49]. & & & \\
\hline
\end{tabular}




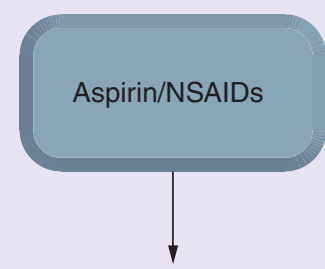

Thromboembolic risk

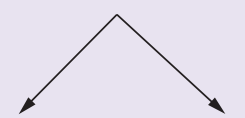

Low
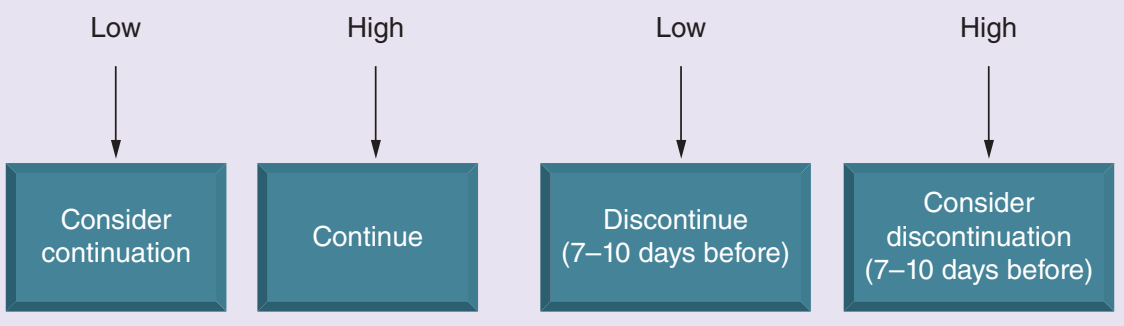

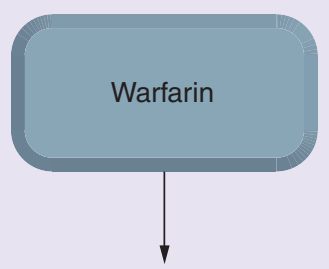

Thromboembolic risk

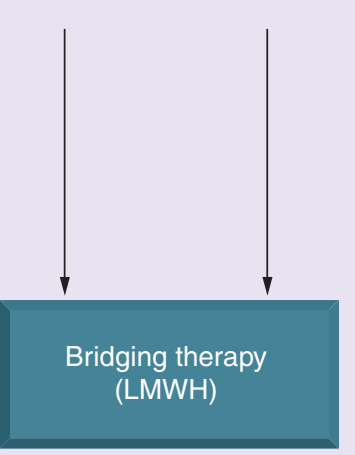

Recommendation based on expert opinion

Figure 1. Management of antithrombotic medication in elective endoscopic placement of gastrostomy for levodopa-carbidopa intestinal gel [40]. Management must be individualized based on risk. In the event of oral anticoagulation, low molecular weight heparins must be used as alternative medication. Risk of bleeding with antiaggregant therapy is greater if patient is receiving clopidogrel.

Further information on equivalences, practical examples and aspects to verify before treatment is given in [50].

\section{Management of antiaggregants/ anticoagulants}

Since PEG is associated with risk of bleeding, antiaggregant/anticoagulant treatment of the patient should be reviewed. Figure 1 provides necessary explanations [40].

\section{- Implementation phase}

Day 1. Patient admission \& placement of nasoduodenal catheter

An optional LCIG response test can be carried out using a nasointestinal catheter before

\section{Table 2. Calculation of levodopa-carbidopa intestinal gel (Duodopa) dose.}

\begin{tabular}{|c|c|}
\hline Recommendations & Examples \\
\hline \multicolumn{2}{|l|}{ Morning dose of LCIG (ml) } \\
\hline Morning dose of levodopa & For example: 100 mg \\
\hline $80 \%$ reduction to decrease hyperkinesia & $100 \times 0.8=80 \mathrm{mg}$ \\
\hline Conversion to $\mathrm{ml}$ of LCIG & $80 \mathrm{mg} / 20 \mathrm{mg} / \mathrm{ml}=4 \mathrm{ml}$ of LClG \\
\hline \multicolumn{2}{|l|}{ Continuous dose of LCIG $(\mathrm{ml} / \mathrm{h})$} \\
\hline Daily dose of levodopa & For example: 1200 mg \\
\hline Subtract from this the morning dose & $1200-100=1100 \mathrm{mg}$ \\
\hline Take $90 \%$ & $1100 \mathrm{mg} \times 90 \%=990 \mathrm{mg}$ \\
\hline Conversion to $\mathrm{ml}$ of LCIG & $990 \mathrm{mg} / 20 \mathrm{mg} / \mathrm{ml}=49.5 \mathrm{ml}$ of LCIG \\
\hline Divided into 16 waking hours & $49.5 \mathrm{ml} / 16 \mathrm{~h}=3.1 \mathrm{ml} / \mathrm{h}$ \\
\hline \multicolumn{2}{|l|}{ Extra dose of LCIG $(\mathrm{ml})$} \\
\hline If morning dose is $>6 \mathrm{ml}$ & Start with an extra dose of $2 \mathrm{ml}$ \\
\hline If morning dose is $<6 \mathrm{ml}$ & Start with an extra dose of $1 \mathrm{ml}$ \\
\hline
\end{tabular}




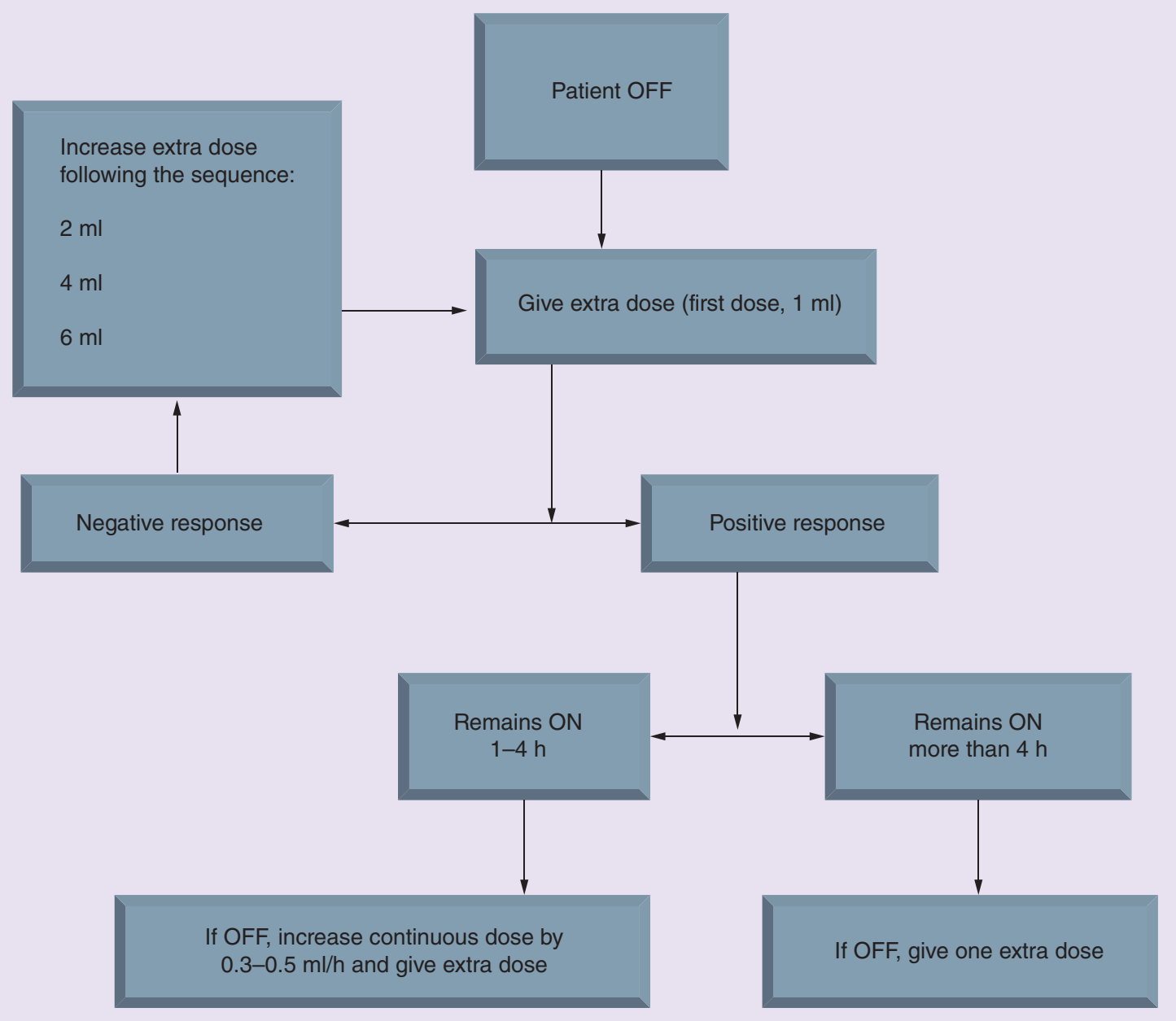

Figure 2. Flow chart for levodopa-carbidopa intestinal gel dose adjustment.

gastrostomy is performed. It can be performed during hospitalization or on an outpatient basis. The test phase allows to monitor response to treatment, observe drug tolerability and familiarize the patient and the caregiver with the pump and infusion system. However, the nasojejunal catheter is sometimes not well tolerated [54]. Fine bore tubes (usually with wire stiffeners) are more flexible, easier to pass and less likely to cause erosions. The most common complications include nasopharyngitis, sinusitis, otitis, tube blockage, misplacement and unwanted removal. For this reason, some authors consider the nasojejunal phase only for patients whose potential clinical benefit is uncertain (e.g., disabling freezing which may not be largely experienced in the 'off' phase, disabling peak-dose or biphasic dyskinesias).

A trained nurse will place the catheter through the nasal route [50] under aseptic conditions (mouth rinsing with $0.12 \%$ chlorhexidine on the evening of the previous day and before functional tests) ensuring that the distal end of the catheter is placed in the duodenum or proximal jejunum. A written informed consent should be obtained.

The procedure may be performed in three ways: by passive migration (most common way, requires 8-24 h); using fluoroscopy; using endoscopy under sedation (for an example of protocol, see [50]). Domperidone (20 mg/8 h) can be administered to facilitate passive migration [55].

\section{Day 2: start of test treatment \& LCIG dose optimization}

Abdominal x-rays should be taken to verify location of the nasoduodenal catheter early in the morning. Adequate catheter location must be ensured (the catheter tip should be at duodeno-jejunal level - ligament of Treitz). If the 
nasointestinal catheter is placed under endoscopic monitoring, abdominal $\mathrm{x}$-rays are not required.

The required LCIG dose will be calculated [56] based on the prior LEDD (mg/day) [49] keeping in mind that $1 \mathrm{ml}$ of LCIG is equivalent to $20 \mathrm{mg}$ of levodopa and $5 \mathrm{mg}$ of carbidopa monohydrate. Three doses must be scheduled: the morning dose; the continuous dose; the extra dose. Table 2 shows how the doses should be scheduled.

Treatment should be started during the morning 'off' period. The pump must be programmed with the previously calculated dose. Five milliliters must be added to the morning dose for purging the nasoduodenal catheter. The extension and connector should be purged, and the catheter should be attached to the pump to start LCIG administration [50]. The nurse and the neurologist will work together to see the response to medication, monitoring the duration of 'on' time, 'off' time and time with dyskinesia. The dose will be adjusted to optimize treatment response. Figure 2 illustrates dose adjustments (P).

Infusion is usually maintained during the day (approximately 12-16 h), but an off-label 24-h administration may be beneficial in specific cases (sleep disorder for akinesia, severe morning akinesia) [57,58]. The LEDD on LCIG is usually similar to the previously received oral LEDD [5].

Day 3 ( $\&$ all other days until PEG). Treatment optimization \& decision to perform PEG During the optimization phase, the following parameters must be defined: the morning dose (the minimum well-tolerated dose required to enter the 'on' state in the shortest time possible), the continuous dose that allows for the longest 'on' time without disabling on-dyskinesia, and the extra dose that allows for quick alleviation of acute 'off' symptoms.

It is essential that the caregiver is trained on pump management and is familiar with symptoms and their causes. Detailed description of the nursing procedures for these days can be found in [50].

The final decision to perform PEG will be jointly taken by the neurologist, nurse, patient and caregiver based on the response, tolerability and undesirable effects. The following should be considered:

- Objective improvement according to the neurologist, including daily 'on' time increase, 'off' time reduction and improvement in dyskinesia duration and severity;

- Improvement in motor symptoms and at least some nonmotor symptoms (pain, sleep, mood, sensory and gastrointestinal symptoms), overall improvement and increase in patient autonomy (E);

- Subjective improvement according to expectations reported by the patient;

- Improvement according to expectations, reported by the caregiver;

- Good treatment tolerability.

\section{Table 3. Complications related to percutaneous endoscopic gastrostomy.}

\begin{tabular}{|c|c|c|c|}
\hline Complication & Predisposing factors & Prevention & $\begin{array}{l}\text { Recommended therapy (based on experts' } \\
\text { experience) }\end{array}$ \\
\hline Pain in abdominal wall & & $\begin{array}{l}\text { Hygiene } \\
\text { Adequate performance of the } \\
\text { procedure }\end{array}$ & $\begin{array}{l}\text { Rule out other complications } \\
\text { Treat with analgesics }\end{array}$ \\
\hline $\begin{array}{l}\text { Benign } \\
\text { pneumoperitoneum }\end{array}$ & Any procedure & Maximum gastric cavity inflation & None in the absence of other clinical signs \\
\hline Paralytic ileus & Gastroparesis & & NGC placement in severe cases only \\
\hline Bleeding & $\begin{array}{l}\text { Antithrombotic therapy } \\
\text { Coagulation defect }\end{array}$ & Adequate medication adjustment & $\begin{array}{l}\text { If bleeding in tract, transient strong } \\
\text { adjustment of gastrostomy catheter } \\
\text { If subcutaneous or rectus abdominis } \\
\text { hematoma, nothing (self-limited) }\end{array}$ \\
\hline Lung aspiration & $\begin{array}{l}\text { Greater risk of aspiration } \\
\text { pneumonia in PEG patients? }\end{array}$ & & Antibiotic therapy if pneumonia \\
\hline Peritonitis & Poor technique & $\begin{array}{l}\text { Correctly performed endoscopic } \\
\text { procedure }\end{array}$ & Laparotomy \\
\hline
\end{tabular}


DRUG EVALUATION Santos García, Martínez Castrillo, Puente Périz et al.

\section{Table 4. Management of complications related to gastrostomy catheter.}

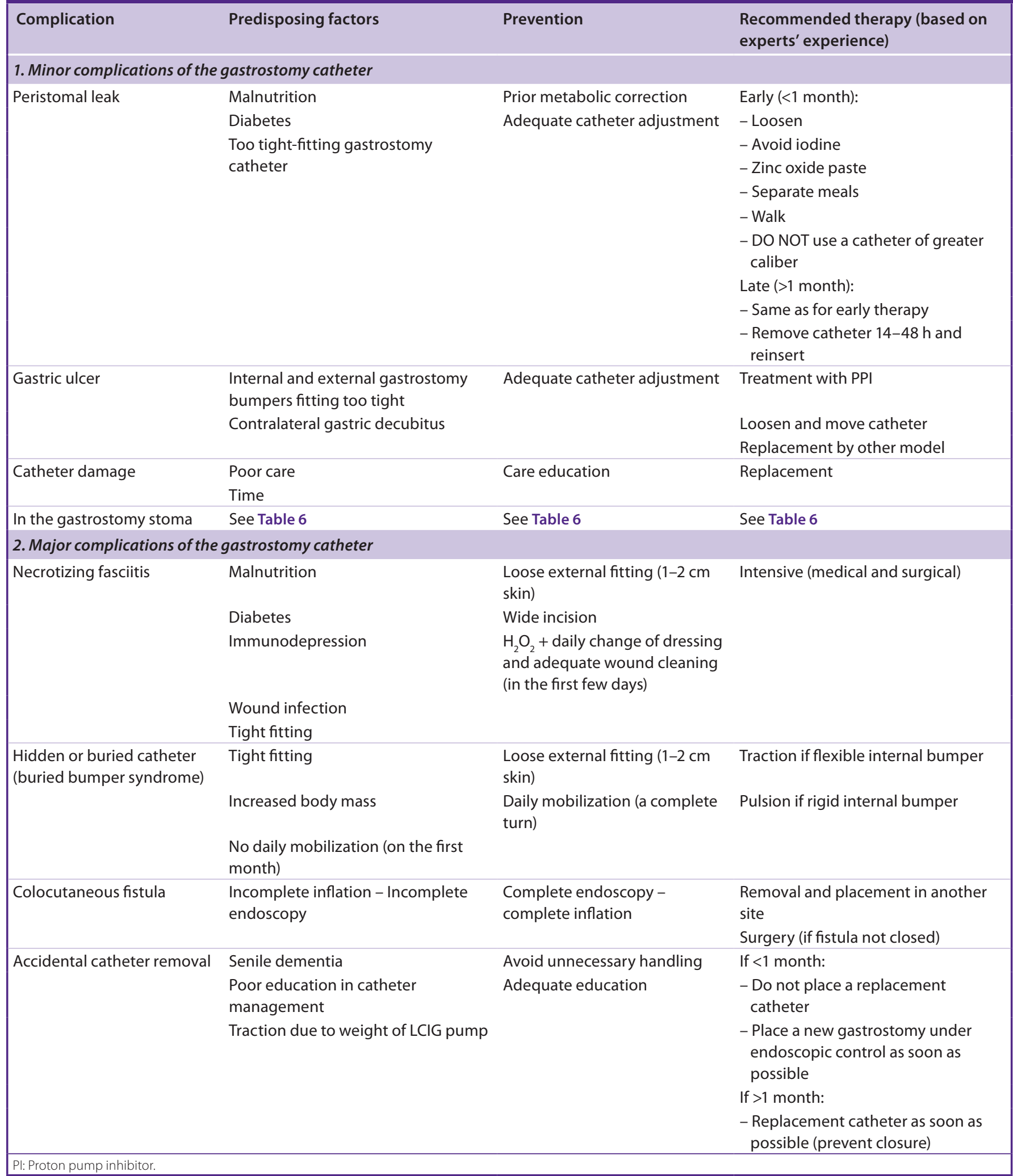




\section{- Phase of percutaneous endoscopic gastrostomy}

In most cases, PEG is performed during patient admission. Alternatively, patient may be discharged on LCIG administered through nasojejunal catheter and attend the endoscopy department on a scheduled date.

Before PEG is performed, preoperative tests (laboratory tests including coagulation, ECG and chest x-ray) and assessment by an anesthesiologist may be required. Patient preparation before surgery include fasting for $8 \mathrm{~h}$, mouth and throat preparation (including denture removal), insertion of a peripheral venous line and administration of prophylactic antibiotics [50]. Patients with LCIG delivered through a gastrointestinal catheter arrive to the endoscopy/operating room with the pump functioning. Before the procedure is started, infusion is stopped and the catheter is removed.

The PEG procedure includes surgical field preparation, anesthetic sedation, high gastrointestinal endoscopy, trans-illumination, puncture under endoscopic monitoring, guidewire recovery through the mouth, insertion of the gastrostomy catheter through the mouth, retainer adjustment, insertion of enteral catheter through the gastrostomy catheter and its conduction until the distal part of the duodenum. Detailed description of the PEG procedure and contraindications for PEG are provided in [50].

Once gastrostomy is performed and the pump is attached, the patient normally remains under observation before being transferred to the hospital ward. It is recommended that the patient

Table 5. Management of complications related to the gastroduodenojejunal catheter.

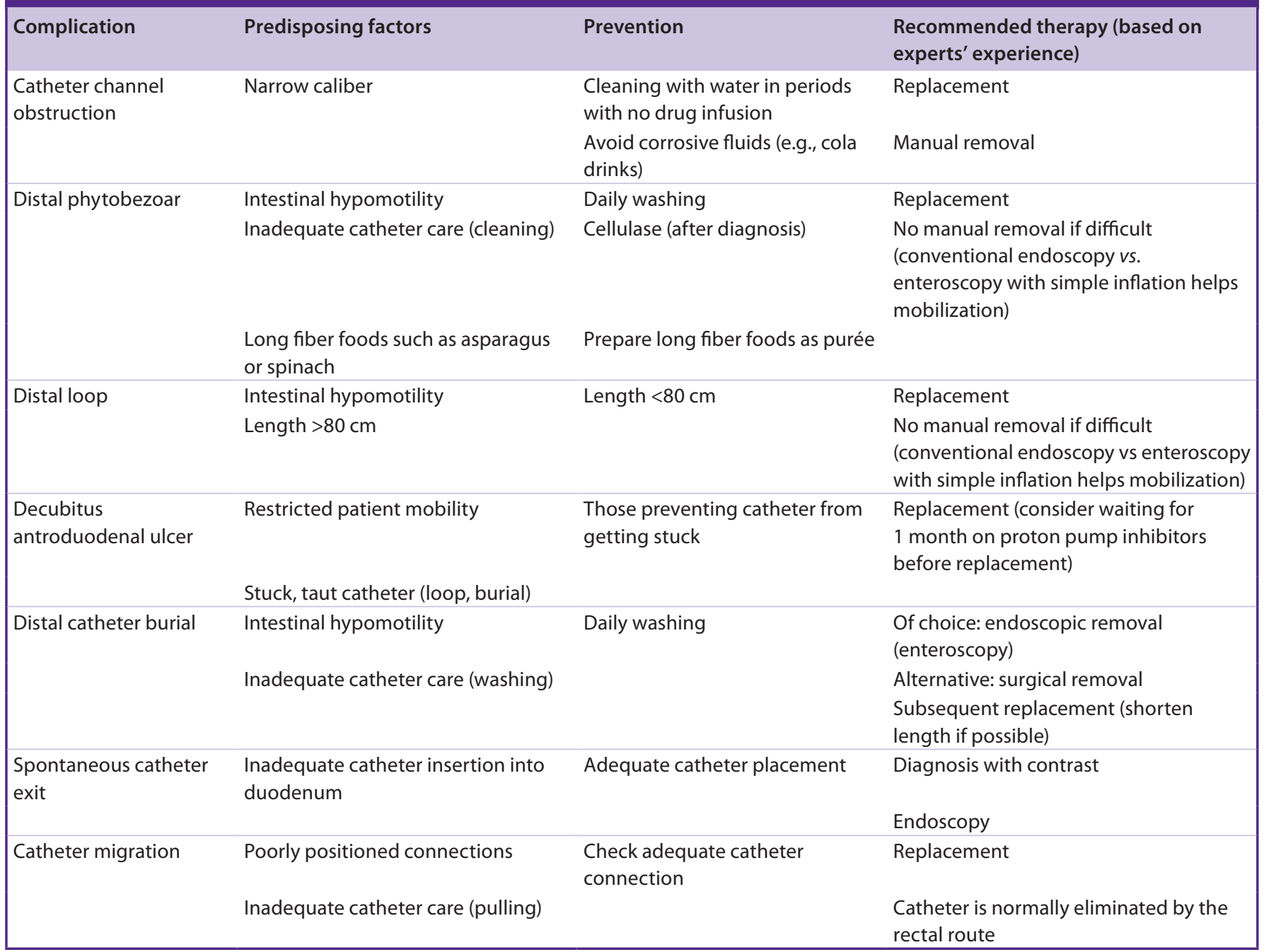


Table 6. Management of complications related to the stoma.

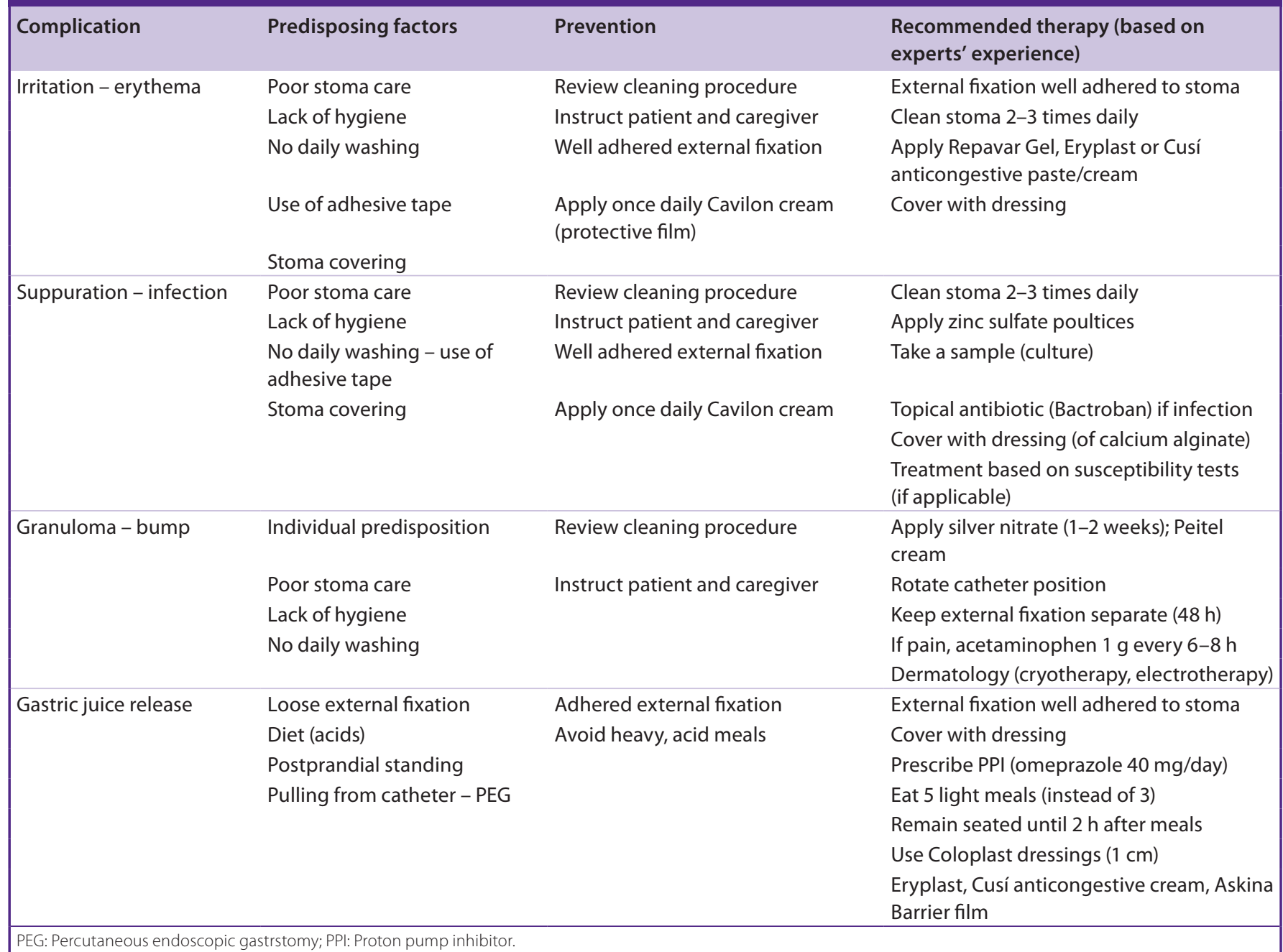

stays at the hospital for $24-48 \mathrm{~h}$ to monitor and treat potential complications, adjust dose and watch response to intestinally delivered medication and educate the patient/caregiver on stoma and device care and pump management $(\mathrm{P})$. Further details nursing management in the postPEG process can be found in [50]. Table 3 shows some potential complications related to PEG.

Alternative outpatient models for PEG have been reported [59]. Patients can undergo endoscopic PEG-J placement as outpatients and be discharged $1-2 \mathrm{~h}$ after the placement is confirmed. The patients are provided with standard postinsertion monitoring information and assessed by the gastroenterologist on the following day. Some authors suggest that the patients return to the clinic off medication 2 weeks later for starting LCIG. LCIG can be initiated and titrated as daytime monotherapy or as adjunctive therapy with other anti-PD medications [60].
- Days subsequent to PEG (until discharge) Potential complications should be monitored. If no complication occurs, patient may be discharged 24-48 h after PEG surgery. Nursing management of patients is of paramount importance [50].

The following should be done before discharge:

- Appoint the next visit with the neurologist;

- Provide material about LCIG to the patient/ caregiver;

- Provide an alternative oral levodopa regimen to use in case of pump failure;

- Ensure contact for potential complications.

- Follow-up phase

Follow-up by the gastroenterologist 
Often scheduled follow-up for regular patient monitoring will not be required. However, a gastroenterologist should be available to resolve potential complications. Table 4, 5 \& 6 show the management of complications related to the gastrostomy and gastroduodenojejunal catheters [50].

The authors consider that the whole system (gastrostomy and gastro-duodenojejunal catheters) should be changed every $1.5-2$ years, unless complications occur earlier (E) [50].

\section{Follow-up by the neurologist}

Monitoring frequency may vary depending on response to medication, tolerability and/or undesirable effects. We recommend to evaluate patients at least $48 \mathrm{~h}$ after discharge, after 15 days, after 1 month and every several months thereafter (E). In stable patients with good treatment response, controls every 3-4 months are recommended (E).

Regular outpatient monitoring should include:

- Motor examination (Hoehn and Yahr and UPDRS);

- Assessment of the need for complete and/or general neurological examination based on patient-reported symptoms (e.g., if lower limb paresthesia or weakness is reported, polyneuropathy should be ruled out);

- Review of dosage and need for adjustment based on patient response (a diary of fluctuations may be provided), degree of satisfaction, tolerability and undesirable effects. Dose adjustments should be made gradually and always taking equivalences into account (0.3-0.5 $\mathrm{ml}$ in continuous dosing; 0.5-1 $\mathrm{ml}$ in morning or extra doses) [50]. In patients on an off-label $24-\mathrm{h}$ infusion, it is important to monitor undesirable effects because they may receive doses higher than $2000 \mathrm{mg}$ of levodopa daily [61];

- Progression of nonmotor symptoms. Specifically, cognitive changes over time would be determinant in patients on long-term treatment (years) with LCIG when deciding whether to continue therapy (E). Whenever possible, try to establish which symptoms are due to disease progression and which ones are influenced by the dopaminergic therapy, and to what extent;

- Presence of undesirable effects or symptoms requiring dose reduction (dizziness, drowsiness, psychotic symptoms, disabling dyskinesia, weight loss, among others);

- Weight control. Patients should be educated to control their weight. In case of significant weight loss, the patient should be referred to a nutritionist;

- Control of the stoma (rule out infection, granulomas, among others);

\section{Box 3. Potential complication of levodopa-carbidopa intestinal gel therapy.}

- Behavioral symptoms or psychotic conditions with delusions, hallucinations (mainly visual), behavioral disorders, impulse control disorder, among others $[64,65]$. It is important to monitor patient's mood because, although no causal relationship is established, at least five cases of suicide have been reported in patients on LCIG treatment [66-69]

- Cognitive changes, with attention problems, episodes of disorientation, confusion, among others [64]

- Autonomic symptoms such as dizziness, hypotension, tachycardia, among others [35]

- Excessive daytime drowsiness (which has negative motor impact and increases risk of falls), sudden episodes of sleep during the day, bewilderment or a sensation of numbness, nausea, vomiting, gastrointestinal intolerance, among others [64]

- Weight loss, sometimes occurring even in patients with a good appetite. In these cases, nutritional status of the patient should be verified (laboratory tests, liver profile, vitamin $B_{12}$ and folate levels, proteins) [70], supplements should be given if required, and patient should be referred to a nutritionist for weight control and prescription of appropriate supplements

- Symptoms suggesting peripheral neuropathy. There have been several reports of Guillain-Barré syndrome [5,71] axonal polyneuropathy or other complications in the setting of deficiency of vitamin $B_{12}$ or other B vitamins [62,70-76]. In the event of acute or subacute and moderateto-severe clinical symptoms, patients should be admitted to hospital and etiology should be established, discontinuing the treatment if required. In case of milder and chronic cases the need for stopping the infusion or decreasing the dose should be assessed (Figure 3) [62]

- Worsening of dyskinesia, which should prompt identification of its timing and potential relationship to the doses. Reduction of continuous dose (or extra or morning doses if dyskinesia occurs after they are administered) will often be required. Some patients may experience severe dyskinesia after the pump is switched off at night (biphasic). In this case, administration of an extra dose shortly before the pump is switched off may be beneficial (E)

CIG: Levodopa-carbidopa instestinal gel 


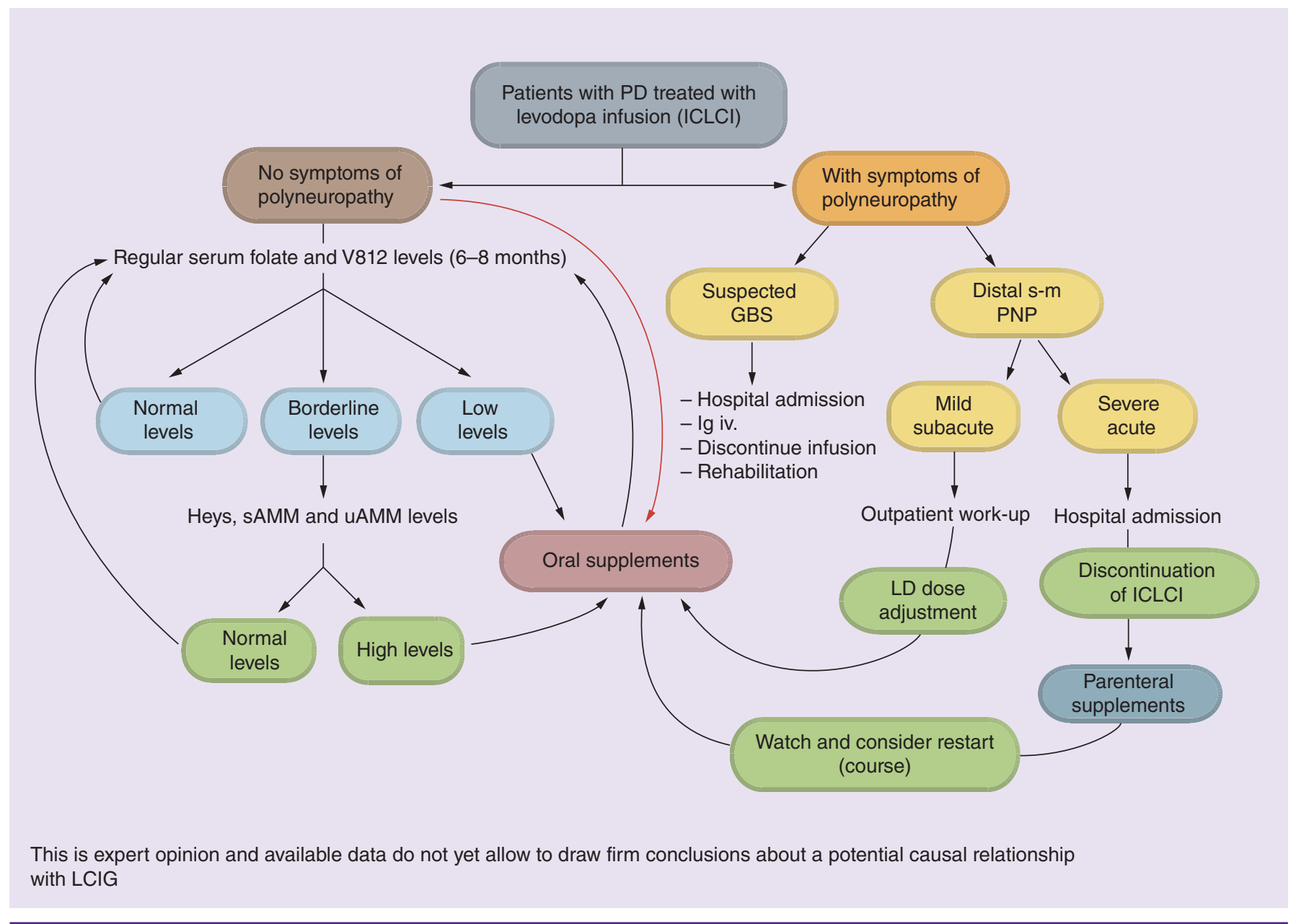

Figure 3. Preventive and therapeutic management for polyneuropathy occurring in patients treated with levodopa-carbidopa intestinal gel [62].

GBS: Guillain-Barré syndrome; Hcys: Homocysteine; LD: Levodopa; PD: Parkinson's disease; PNP: Polyneuropathy; sMMA: Serum methylmalonic acid; uMMA: Urinary methylmalonic acid.

- Control of the infusion system;

- Regular laboratory controls including serum levels of vitamin $\mathrm{B}_{12}$ and folate. An electroneurography could be indicated in some cases. Treat with oral supplements if needed [62].

Follow-up by the nurse

General objectives [50]:

- To provide direct and continued personalized care to the patient/caregiver;

- To establish a therapeutic relationship where the patient plays an active role in his/her treatment process;
- To educate and train the patient/caregiver in preventing and managing local complications;

- To assess the patient, to record the collected data and to report relevant incidents to the neurologist in charge.

Specific objectives:

- To verify adequate management of the infusion system;

- To monitor the stoma status and to manage any complications that may arise (Table 6);

- To resolve any doubt or problem that a patient may have; 
- To improve the quality of life of patients/caregivers by providing them adequate education.

\section{When should LCIG be discontinued?}

In general, stopping the treatment should be considered when the benefits of the therapy are low or the risks outweigh the benefits. In our opinion, this decision must be justified and discussed with the patient and family (E). Stopping LCIG should be considered in the following circumstances:

- The patient becomes bedridden in the best 'on' state (Hoehn and Yahr Stage 5);

- The patient develops dementia with dependency for activities of daily living;

- The patient develops frequent and/or disabling complications related to the treatment and/or to the system that cannot be adequately controlled [29,63];

- The patient develops absolute dependence due to other comorbidities (arthrosis, diabetes, among others).

\section{Management of complications}

It is important to educate patients to recognize urgent complications (i.e., fever, vomiting, abdominal pain or exit of the gastrostomy set), which should be promptly treated in an emergency room, and nonurgent complications (i.e., pump/catheter malfunction or stoma leak), which should be assessed at an outpatient clinic. Sometimes patients should be switched to oral levodopa until such assessment occurs.

LCIG is usually well tolerated and causes no more drug-related complications than oral levodopa [24]. It is important to identify complications that may improve upon dose reduction (additional symptomatic treatment may sometimes be required). Potential complications are described in Box 3 \& Figure 3 [1,5,35,62,64-76].

Any case of potential complication related to the gastrostomy and enteral catheter should be communicated to the gastroenterologist and/or specialized nurse. Complications related to the parts of the device and stoma should be managed by the specialized nurse.

\section{Future perspective}

- It is possible that in the following years patients may be selected for LCIG treatment in earlier stages of the disease;

- LCIG treatment implementation might be simplified, omitting nasojejunal test phase and proceeding directly to outpatient optimization and PEG surgery, thus switching rapidly from oral to enteral levodopa treatment (equivalent dose);

- We hope that the future will bring more comparative studies of different treatments of advances PD (surgery, enteral levodopa or apomorphine infusions) in settings of daily clinical practice;

- We believe that patients' profiles for particular treatments of advanced PD will be better defined, ensuring maximal benefit from each treatment;

- It is possible that other, less invasive routes of administration for levodopa delivery (i.e., subcutaneous) will be available;

- It is essential to carry out studies designed to compare motor complications development and disease progression between patients receiving LCIG since early stages of the disease and patients receiving conventional oral medication.

\section{Conclusion}

LCIG is an effective treatment in patients with advanced PD that allows for improving their motor condition, nonmotor symptoms, quality of life and degree of autonomy. In order to achieve the expected result, good candidate selection and adequate monitoring are required. Treatment and patient care should be multidisciplinary, with active involvement of the neurologist, gastroenterologist and nursing staff. Patient and caregiver education is essential. Use of protocols and application of the knowledge provided in clinical management guidelines such as these will allow therapeutic success.

Financial \& competing interests disclosure

The drafting committee has received support from AbbVie Spain, S.L.U. for preparing this manuscript. The authors have no other relevant affiliations or financial involvement with any organization or entity with a financial interest in or financial conflict with the subject matter or materials discussed in the manuscript apart from those disclosed.

Writing assistance was utilized in the production of this manuscript. Content Ed Net (Madrid, Spain) has collaborated in writing of the manuscript. 


\section{Disclosure}

In addition to the peer-review process, with the author's consent, the manufacturer of the product discussed in this article was given the opportunity to review the manuscript for factual accuracy. Changes were made by the author at their discretion and based on scientific or editorial merit only. The author maintained full control over the manuscript, including content, wording and conclusions.

\section{References}

Papers of special note have been highlighted as: - of interest; $\bullet$ of considerable interest

1 Duodopa. Summary of Product Characteristics www.medicines.org.uk/emc/medicine/20786

2 Nyholm D, Askmark H, Gomes-Trolin C et al. Optimizing levodopa pharmacokinetics: intestinal infusion versus oral sustainedrelease tablets. Clin. Neuropharmacol. 26(3), 156-163 (2003).

3 Nyholm D, Nilsson Remahl AI, Dizdar N et al. Duodenal levodopa infusion monotherapy vs oral polypharmacy in advanced Parkinson disease. Neurology 64(2), 216-223 (2005).

4 Palhagen SE, Dizdar N, Hauge T et al. Interim analysis of long-term intraduodenal levodopa infusion in advanced Parkinson disease. Acta Neurol. Scand. 126(6), e29-e33 (2012).

5 Antonini A, Isaias IU, Canesi M et al. Duodenal levodopa infusion for advanced Parkinson's disease: 12 month treatment outcome. Mov. Disord. 22(8), 1145-1149 (2007).

6 Antonini A, Yegin A, Preda C et al. Global long-term study on motor and non-motor symptoms and safety of levodopa-carbidopa intestinal gel in routine care of advanced Parkinson's disease patients; 12 month interim outcomes. Parkinsonism Relat. Disord. 21(3), 231-235 (2015).

7 Devos D, French DSG. Patient profile, indications, efficacy and safety of duodenal levodopa infusion in advanced Parkinson's disease. Mov. Disord. 24(7), 993-1000 (2009).

8 Eggert K, Schrader C, Hahn M et al. Continuous jejunal levodopa infusion in patients with advanced parkinson disease: practical aspects and outcome of motor and non-motor complications. Clin. Neuropharmacol. 31(3), 151-166 (2008).

9 Elia AE, Dollenz C, Soliveri P, Albanese A. Motor features and response to oral levodopa in patients with Parkinson's disease under continuous dopaminergic infusion or deep brain stimulation. Eur. J. Neurol. 19(1), 76-83 (2012).

10 Fernandez HH, Standaert DG, Hauser RA et al. Levodopa-carbidopa intestinal gel in advanced Parkinson's disease: final 12 month, open-label results. Mov. Disord. 30(4), 500-509 (2015).

- To date, this is the largest published international, prospective, open-label levodopa-carbidopa intestinal gel (LCIG) study. LCIG was generally well tolerated and demonstrated clinically significant improvements in motor function, daily activities and HRQoL sustained over 54 weeks.

11 Fernandez HH, Vanagunas A, Odin P et al. Levodopa-carbidopa intestinal gel in advanced Parkinson's disease open-label study: interim results. Parkinsonism Relat. Disord. 19(3), 339-345 (2013).

12 Isacson D, Bingefors K, Kristiansen IS, Nyholm D. Fluctuating functions related to quality of life in advanced Parkinson disease: effects of duodenal levodopa infusion. Acta Neurol. Scand. 118(6), 379-386 (2008).

13 Kurth MC, Tetrud JW, Tanner CM et al. Double-blind, placebo-controlled, crossover study of duodenal infusion of levodopa/ carbidopa in Parkinson's disease patients with 'on-off' fluctuations. Neurology 43(9), 1698-1703 (1993).

14 Martinez-Martin P, Reddy P, Katzenschlager $\mathrm{R}$ et al. EuroInf: a multicenter comparative observational study of apomorphine and levodopa infusion in Parkinson's disease. Mov. Disord. 30(4), 510-516 (2015).

- Based on this open-label, nonrandomized, comparative study, both LCIG $(n=44)$ and apomorphine infusion therapy $(n=43)$ appear to provide a robust improvement in motor symptoms, motor complications, quality-of-life and some nonmotor symptoms.

15 Meppelink AM, Nyman R, Van Laar T, Drent M, Prins T, Leenders KL.

Transcutaneous port for continuous duodenal levodopa/carbidopa administration in Parkinson's disease. Mov. Disord. 26(2), 331-334 (2011).

16 Merola A, Zibetti M, Angrisano S, Rizzi L, Lanotte M, Lopiano L. Comparison of subthalamic nucleus deep brain stimulation and Duodopa in the treatment of advanced Parkinson's disease. Mov. Disord. 26(4), 664-670 (2011).
17 Munro Neville A, Parsons RW, Askmark H, Nyholm D. Treatment of advanced Parkinson's disease with levodopa/carbidopa intestinal gel is associated with improvements in Hoehn and Yahr stage. Parkinsonism Relat. Disord. 18(5), 686-687 (2012).

18 Nilsson D, Hansson LE, Johansson K, Nystrom C, Paalzow L, Aquilonius SM. Long-term intraduodenal infusion of a water based levodopa-carbidopa dispersion in very advanced Parkinson's disease. Acta Neurol. Scand. 97(3), 175-183 (1998).

19 Nilsson D, Nyholm D, Aquilonius SM. Duodenal levodopa infusion in Parkinson's disease - long-term experience. Acta Neurol. Scand. 104(6), 343-348 (2001).

20 Nyholm D. Duodopa(R) treatment for advanced Parkinson's disease: a review of efficacy and safety. Parkinsonism Relat. Disord. 18(8), 916-929 (2012).

21 Nyholm D, Johansson A, Lennernas H, Askmark H. Levodopa infusion combined with entacapone or tolcapone in Parkinson disease: a pilot trial. Eur. J. Neurol. 19(6), 820-826 (2012).

22 Nyholm D, Klangemo K, Johansson A. Levodopa/carbidopa intestinal gel infusion long-term therapy in advanced Parkinson's disease. Eur. J. Neurol. 19(8), 1079-1085 (2012).

23 Nyholm D, Lewander T, Johansson A, Lewitt PA, Lundqvist C, Aquilonius SM. Enteral levodopa/carbidopa infusion in advanced Parkinson disease: long-term exposure. Clin. Neuropharmacol. 31(2), 63-73 (2008).

24 Olanow CW, Kieburtz K, Odin P et al. Continuous intrajejunal infusion of levodopa-carbidopa intestinal gel for patients with advanced Parkinson's disease: a randomised, controlled, double-blind, doubledummy study. Lancet Neurol. 13(2), 141-149 (2014).

- To date, this is the only randomized, double-blind, double-dummy, double-titration trial (class I study) demostrating the benefit of LCIG in the control of motor complications in advanced Parkinson's disease.

25 Puente V, De Fabregues O, Oliveras C et al. Eighteen month study of continuous intraduodenal levodopa infusion in patients with advanced Parkinson's disease: impact on control of fluctuations and quality of life. 
Parkinsonism Relat. Disord. 16(3), 218-221 (2010).

26 Reddy P, Martinez-Martin P, Rizos A et al. Intrajejunal levodopa versus conventional therapy in Parkinson disease: motor and nonmotor effects. Clin. Neuropharmacol. 35(5), 205-207 (2012).

-• This study demonstrates the positive effects of LCIG on motor and nonmotor symptoms versus the best conventional therapy in daily clinical practice.

27 Santos-Garcia D, Macias M, Llaneza M et al. Experience with continuous levodopa enteral infusion (Duodopa $((\mathrm{R}))$ ) in patients with advanced Parkinson's disease in a secondary level hospital. Neurologia 25(9), 536-543 (2010).

28 Sensi M, Preda F, Trevisani L et al. Emerging issues on selection criteria of levodopa carbidopa infusion therapy: considerations on outcome of 28 consecutive patients. J. Neural. Transm. (Vienna) 121(6), 633-642 (2014).

29 Slevin JT, Fernandez HH, Zadikoff C et al. Long-term safety and maintenance of efficacy of levodopa-carbidopa intestinal gel: an open-label extension of the double-blind pivotal study in advanced Parkinson's disease patients. J. Parkinsons Dis. 5(1), 165-174 (2015).

30 Syed N, Murphy J, Zimmerman T Jr, Mark $\mathrm{MH}$, Sage JI. Ten years' experience with enteral levodopa infusions for motor fluctuations in Parkinson's disease. Mov. Disord. 13(2), 336-338 (1998).

31 Timpka J, Fox T, Fox K et al. Improvement of dyskinesias with 1-dopa infusion in advanced Parkinson's disease. Acta Neurol. Scand. doi:10.1111/ane.12483 (2015) (Epub ahead of print).

32 Antonini A, Fung VS, Boyd JT et al. Effect of levodopa-carbidopa intestinal gel on dyskinesia in advanced Parkinson's disease patients. Mov. Disord. doi:10.1002/ mds.26528 (2016) (Epub ahead of print).

33 Buongiorno $\mathrm{M}$, Antonelli F, Cámara A et al. Long-term response to continuous duodenal infusion of levodopa/carbidopa gel in patients with advanced Parkinson disease: the Barcelona registry. Parkinsonism Relat. Disord. 21(8), 871-876 (2015).

34 Honig H, Antonini A, Martinez-Martin P et al. Intrajejunal levodopa infusion in Parkinson's disease: a pilot multicenter study of effects on nonmotor symptoms and quality of life. Mov. Disord. 24(10), 1468-1474 (2009).

- The first published study (open-label) demostrating the positive effect of LCIG on nonmotor symptoms.
35 Pursiainen V, Lyytinen J, Pekkonen E. Effect of duodenal levodopa infusion on blood pressure and sweating. Acta Neurol. Scand. 126(4), e20-e24 (2012).

36 Zibetti M, Merola A, Ricchi V et al. Long-term duodenal levodopa infusion in Parkinson's disease: a 3 year motor and cognitive follow-up study. J. Neurol. 260 (1), 105-114 (2013).

37 Antonini A, Mancini F, Canesi M et al. Duodenal levodopa infusion improves quality of life in advanced Parkinson's disease. Neurodegener. Dis. 5(3-4), 244-246 (2008).

38 Santos-Garcia D, Sanjurjo LF, Macias M, Llaneza M, Carpintero P, De La FuenteFernandez R. Long-term exposure to duodenal levodopa/carbidopa infusion therapy improves quality of life in relation especially to mobility, activities of daily living, and emotional well-being. Acta Neurol. Scand. 125(3), 187-191 (2012).

39 Daniel SE, Lees AJ. Parkinson's Disease Society Brain Bank, London: overview and research. J. Neural. Transm. Suppl. 39, 165-172 (1993).

40 Committee ASOP, Anderson MA, BenMenachem $T$ et al. Management of antithrombotic agents for endoscopic procedures. Gastrointest. Endosc. 70 (6), 1060-1070 (2009).

41 Kempster PA, Williams DR, Selikhova M, Holton J, Revesz T, Lees AJ. Patterns of levodopa response in Parkinson's disease: a clinico-pathological study. Brain 130 (Pt 8), 2123-2128 (2007).

42 Calandrella D, Romito LM, Elia AE et al. Causes of withdrawal of duodenal levodopa infusion in advanced Parkinson disease. Neurology 84(16), 1669-1672 (2015).

43 Antonini A, Odin P. Pros and cons of apomorphine and L-dopa continuous infusion in advanced Parkinson's disease. Parkinsonism Relat. Disord. 15(Suppl. 4), S97-S100 (2009).

44 Sanchez-Castaneda C, Campdelacreu J, Miro J, Juncadella M, Jauma S, Calopa M. Cognitive improvement after duodenal levodopa infusion in cognitively impaired Parkinson's disease patients. Prog. Neuropsychopharmacol. Biol. Psychiatry 34(1), 250-251 (2010).

45 Catalan MJ, De Pablo-Fernandez E, Villanueva C et al. Levodopa infusion improves impulsivity and dopamine dysregulation syndrome in Parkinson's disease. Mov. Disord. 28(14), 2007-2010 (2013).

46 Todorova A, Samuel M, Brown RG, Chaudhuri KR. Infusion therapies and development of impulse control disorders in advanced Parkinson's disease: clinical experience after 3 years' follow-up. Clin. Neuropharmacol. 38(4), 132-134 (2015).

47 Volkmann J, Albanese A, Antonini A. Selecting deep brain stimulation or infusion therapies in advanced Parkinson's disease: an evidence-based review. J. Neurol. 260(11), 2701-2714 (2013).

48 Odin P, Ray Chaudhuri K et al. Collective physician perspectives on non-oral medication approaches for the management of clinically relevant unresolved issues in Parkinson's disease: consensus from an international survey and discussion program. Parkinsonism Relat. Disord. 21(10), 1133-1144 (2015).

- Recommendations on the management of Parkinson's disease refractory to oral/ transdermal therapies involving 103 experts from 13 countries overseen by an International Steering Committee (ISC) of 13 movement disorder specialists.

49 Tomlinson CL, Stowe R, Patel S, Rick C, Gray R, Clarke CE. Systematic review of levodopa dose equivalency reporting in Parkinson's disease. Mov. Disord. 25(15), 2649-2653 (2010).

50 Extracts from Practical guidelines for the treatment of advanced Parkinson's disease using continuousintestinal infusion of levodopa/carbidopa (Duodopa ${ }^{\circledR}$ ). www.contentednet.com/projects/duodopaguidelines/

51 Santos-Garcia D, Anon MJ, Fuster-Sanjurjo L, De La Fuente-Fernandez R. Duodenal levodopa/carbidopa infusion therapy infusion in patients with advanced Parkinson'sdisease leads to improvement in caregivers' stress and burden. Eur. J. Neurol. 19(9), 1261-1265 (2012).

52 Solla P, Fasano A, Cannas Aet al. Dopamine agonist withdrawal syndrome (DAWS) symptoms in Parkinson's disease patients treatedwith levodopa-carbidopa intestinal gel infusion. Parkinsonism Relat.Disord. 21(8), 968-971 (2015).

53 Meiler B, Andrich J, Muller T. Rapid switch from oral antiparkinsonian combination drug therapy to duodenal levodopa infusion. Mov. Disord. 23 (1), 145-146 (2008).

54 Pearce CB, Duncan HD. Enteral feeding. Nasogastric, nasojejunal, percutaneous endoscopic gastrostomy, or jejunostomy: its indications and limitations. Postgrad. Med.J. 78(918), 198-204 (2002).

55 Pedersen SW, Clausen J, Gregerslund MM. Practical guidance on how to handle levodopa/carbidopa intestinal gel therapy of 
advanced PD in a movement disorder clinic. Open Neurol. J. 6, 37-50 (2012).

56 Wenzelburger R, Zhang BR, Pohle S et al. Force overflow and levodopa-induced dyskinesias in Parkinson's disease. Brain 125(Pt 4), 871-879 (2002).

57 Chang FC, Tsui DS, Mahant N et al. $24 \mathrm{~h}$ Levodopa-carbidopa intestinal gel may reduce falls and "unresponsive" freezing of gait in Parkinson's disease. Parkinsonism Relat. Disord. 21(3), 317-320 (2015).

- A pilot study with five patients indicating that 24-h LCIG therapy may reduce levodopa 'unresponsive' freezing of gate and associated falls.

58 Nyholm D, Jansson R, Willows T, Remahl IN. Long-term 24-hour duodenal infusion of levodopa: outcome and dose requirements. Neurology 65(9), 1506-1507 (2005).

59 Fasano A, Liu LW, Poon YY, Lang AE. Initiating intrajejunal infusion of levodopa/ carbidopa intestinal gel: an outpatient model. Mov. Disord. 30 (4), 598-599 (2015).

- This article describes a novel and interesting outpatient model for initiating intrajejunal infusion of LCIG.

60 Lew MF, Slevin JT, Krüger R et al. Initiation and dose optimization for levodopa-carbidopa intestinal gel: insights from Phase 3 clinical trials. Parkinsonism Relat. Disord. 21(7), 742-748 (2015).

61 Jugel C, Ehlen F, Taskin B, Marzinzik F, Muller T, Klostermann F. Neuropathy in Parkinson's disease patients with intestinal levodopa infusion versus oral drugs. PLoS ONE 8(6), e66639 (2013).
62 Santos-Garcia D, De La Fuente-Fernandez R, Valldeoriola F et al. Polyneuropathy while on duodenal levodopa infusion in Parkinson's disease patients: we must be alert. J. Neurol. 259(8), 1668-1672 (2012).

63 Lang AE, Rodriguez RL, Boyd JT et al. Integrated safety of levodopa-carbidopa intestinal gel from prospective clinical trials. Mov. Disord. doi:10.1002/mds.26485 (2015) (Epub ahead of print).

64 Santos-Garcia D, De Deus T, Lopez-Pazos E et al. Management of complications related to intraduodenal infusion of levodopa/carbidopa in patients with Parkinson's disease. Rev. Neurol. 58(11), 505-515 (2014).

65 Salomone G, Marano M, Di Biase L, Melgari JM, Di Lazzaro V. Dopamine dysregulation syndrome and punding in levodopacarbidopa intestinal gel (LCIG) infusion: a serious but preventable complication. Parkinsonism Relat. Disord. 21(9), 1124-1125 (2015).

66 Santos-Garcia D, Macias M, Llaneza M, Aneiros A. Suicide following duodenal levodopa infusion for Parkinson's disease. Mov. Disord. 24(13), 2029-2030 (2009).

67 Flisar D AA. Suicide in a PD patient treated with Duodopa - second case. WFN 18, S129 (2011).

68 Fernandez HH, Standaert DG, Chatamra K, Benesh JA. Reply to letter: suicide in Parkinson's disease patients treated with levodopa-carbidopa intestinal gel. Mov. Disord. 30(10), 1435-1436 (2015).

69 Zorko N, Kojovic M, Flisar D, Pirtosek Z, Kramberger MG. Suicide in Parkinson's disease patients treated with levodopa- carbidopa intestinal gel. Mov. Disord. 30 (10), 1434-1435 (2015).

70 Santos-Garcia D, Macias M, Llaneza M, Grande M, De La Fuente-Fernandez R. Serum vitamin $\mathrm{B}(12)$ and folate levels in Parkinson's disease patients treated with duodenal levodopa infusion. Mov. Disord. 26(3), 558-559 (2011).

71 Onofrj M, Bonanni L, Cossu G, Manca D, Stocchi F, Thomas A. Emergencies in parkinsonism: akinetic crisis, life-threatening dyskinesias, and polyneuropathy during L-Dopa gel treatment. Parkinsonism Relat. Disord. 15(Suppl. 3), S233-S236 (2009).

72 Manca D, Cossu G, Murgia D et al. Reversible encephalopathy and axonal neuropathy in Parkinson's disease during duodopa therapy. Mov. Disord. 24(15), 2293-2294 (2009).

73 Skodda S, Muller T. Refractory epileptic seizures due to vitamin B6 deficiency in a patient with Parkinson's disease under duodopa(R) therapy. J. Neural. Transm. (Vienna) 120(2), 315-318 (2013).

74 Urban PP, Wellach I, Faiss $S$ et al. Subacute axonal neuropathy in Parkinson's disease with cobalamin and vitamin B6 deficiency under duodopa therapy. Mov. Disord. 25(11), 1748-1752 (2010).

75 Valldeoriola FCA. Infusión intraduodenal de Duodopa. Rev. Neurol. 51, 41-48 (2010).

76 Uncini A, Eleopra R, Onofrj M. Polyneuropathy associated with duodenal infusion of levodopa in Parkinson's disease: features, pathogenesis and management. J. Neurol. Neurosurg. Psychiatry 86(5), 490-495 (2015). 